\title{
LA SISTEMATIZACIÓN DE EXPERIENCIAS. ENTREVISTA CON ÓSCAR JARA HOLLIDAY
}

\section{EXPERIENCE SYSTEMATIZATION. INTERVIEW WITH ÓSCAR JARA HOLLIDAY}

Fecha de recepción: 09/01/2019

Óscar Jara Holliday*

Fecha de aceptación: 23/04/2019

Resumen: En la presente entrevista, el destacado educador popular y sociólogo peruanocostarricense, Dr. Óscar Jara Holliday, destaca las particularidades de la sistematización de experiencias (SE) como una perspectiva para la acción social, la reflexión dialógica y la crítica de sus participantes acerca de sus experiencias vividas, así como sus potencialidades para el planteamiento o solución de problemáticas sociales, políticas, económicas, etc., que involucran un grupo social en específico. En palabras de Jara Holliday, la SE busca responder a las preguntas: “¿qué estamos haciendo?, ¿cómo lo estamos haciendo?, ¿para qué lo estamos haciendo? Entonces, sistematización de experiencias primero es un proceso, es un enfoque, y es una propuesta metodológica que parte participativamente de los propios sujetos que viven la propia experiencia, son los principales sujetos protagonistas de la sistematización. Segundo, la sistematización no es para reconstruir solo lo que se ha vivido, sino para iluminar lo que queremos hacer".

Palabras claves: entrevista; sistematización de experiencias; educación; metodología; Óscar Jara Holliday.

Abstract: In the preset interview, the salient Peruvian-Costa Rican popular educator and sociologist, Dr. Óscar Jara Holliday, highlights the particularities of experience systematization (ES) as a perspective for social action, the dialogic and the critical reflection of its participants about their lived experiences, as well as its potentialities to approach or solve social, political, economic issues, etc., that involve a specific social group. In Jara Holliday's words, the ES looks to answer the following questions: "What are we doing? How are we doing

* Peruano-costarricense. Sociólogo y Educador Popular. Director General del Centro de Estudios y Publicaciones Alforja en Costa Rica. Coordinador del Programa Latinoamericano de Apoyo a la Sistematización de Experiencias del Consejo de Educación Popular de América Latina y El Caribe (CEAAL). Correo electrónico: oscar@cepalforja.org Entre sus obras destacan: Educación popular: la dimensión de la acción política (1981); Los desafíos de la eduación popular (1984); Aprender desde la práctica (1989); Para sistematizar experiencias (1994); Apropiarse del futuro (2006); Educación y cambio social en América Latina (2009); y La sistematización de experiencias. Práctica y teoría para otros mundos posibles (2012). Investigador referencia en la teoría y la práctica de la sistematización experiencia, es fundador de la Red Alforja junto a Carlos Núñez, del Imdec de Guadalajara, y Raúl Leis, del Ceaspa de Panamá. Ha sido promotor, coordinador y animador de procesos de educación popular en países de prácticamente todo el orbe, principalmente, en América Latina. Además, durante más de 30 años ha laborado como conferencista en seminarios congresos internacionales relacionados con la educación popular y la sistematización de experiencias. 
it? Why are we doing it? First of all then, experience systematization it's a process, an approach, and a methodological proposal that participatively sets off from the same subjects that live their very own experiences, which are the protagonist subjects of the systematization. Second, the purpose of systematization it's not the reconstruction of the lived, but for the illumination of what we want to do".

Keywords: Interview; Experience Systematization; Education; Methodology; Óscar Jara Holliday.

\section{La sistematización de experiencias}

Revista Perspectivas (RP): Iniciamos con la temática sobre sistematización de experiencias ¿Cómo y cuándo surge la sistematización de experiencias en los procesos de investigación educativa?

Óscar Jara Holliday (OJH): En realidad, rastreando el origen del tema de sistematización de experiencias y por qué se llama así, se genera también una confusión con el uso común que tiene la palabra sistematización, que es ordenar, clasificar, catalogar una diversidad de datos e informaciones que es sistematización de información. Muchas veces se confunde la sistematización de información que se hace en la disciplina con el concepto, pero esta perspectiva es más compleja de cuando hablamos de sistematizar experiencias como procesos de construcción de conocimiento desde la práctica, procesos de reflexión crítica. Entonces, cuando se entiende como sistematización de información nada más, se sugiere una idea acerca de clasificar, de ordenar la experiencia, y no tanto de hacer una reflexión crítica sobre esta. Pensando de dónde es que surge este terreno, en el proceso de construcción de la disciplina del trabajo social en América Latina hubo un proceso en la década de 1960 en lo que era la disciplina de asistencia social -y que después se llamó servicio social-, que estaba más bien orientada con moldes norteamericanos principalmente, incluso había una asociación latinoamericana de asistencia social que era dirigida por una organización estadounidense y esa asociación latinoamericana. Estamos hablando del decenio de 1960 y en esa visión las problemáticas que se trataban eran particulares, personales; normalmente, una mujer era la que trabajaba en relación con esos casos individuales problemáticos.

En la década de 1960, producto de todo lo que implicó el cambio conceptual que crea la revolución cubana, a partir de poder mirar nuestra realidad, la realidad latinoamericana, desde América Latina y romper con los moldes europeos o norteamericanos con los cuales los Estudios Sociales estaban prioritariamente orientados, surge un movimiento que se llama la reconceptualización de las asistencias sociales o experiencias sociales. En 1970 me encontré un texto de Leila Lima presentado en una ponencia realizada en Ecuador en 1971, que se titula más o menos así, La práctica del Trabajo Social 
como fuente de teoría. En ese trabajo, ella argumenta que la gente que hace servicio social -o asistencia social o, llamado ahora, trabajo social- está involucrada con los procesos, con las problemáticas sociales, mucho más que los sociólogos, economistas, historiadores, psicólogos, etc., en la naciente ciencia social latinoamericana que había en el decenio de 1960. Leila subraya que nosotros tenemos un conocimiento que viene de esa práctica y muchas veces esta se nos menosprecia porque no hemos generado una disciplina que nos permita sustentar académicamente el valor de nuestro trabajo y del conocimiento que se produce en la práctica.

Para sustentar nuestra disciplina, Leila menciona la necesidad de producir un conocimiento sistemático, y para producir ese conocimiento sistemático de nuestras experiencias tenemos que sistematizar las experiencias. Este es uno de los primeros documentos, que yo encuentro, donde se habla explícitamente de sistematizar experiencias como reflexión crítica que permite la producción de un conocimiento teórico. A partir de ahí ese término se va generalizando; primero procede del trabajo social, luego se va vinculando con procesos de educación popular y a partir de finales de la década de 1970, en América Latina empezamos a utilizar sistematización de experiencias en el sentido de construir aprendizajes, reflexiones críticas, aprendizajes significativos de las experiencias que realizamos. Esto supone colocar un valor a las experiencias, es decir, que hay un conocimiento que está en las experiencias pero que necesitamos producirlo y crearlo de una manera rigurosa, sistemática.

En última instancia, el surgimiento procede de la idea de valorar las experiencias que realizamos, sobre todo las experiencias sociales, el trabajo con comunidades, con movimientos indígenas, etc., resaltando que esa experiencia es una fuente de aprendizaje. Pero, para sistematizar, necesitamos construir una propuesta, una metodología rigurosa que nos permita producir un conocimiento serio a partir de lo que esas experiencias nos enseñaron. $\mathrm{O}$ sea, no es una cosa que se da automáticamente. Por supuesto que todos aprendemos un poco de lo que nos pasa en la vida cotidiana, pero la idea de sistematizar las experiencias significa, intencionadamente, producir un conocimiento a partir de las experiencias. Y nosotros lo utilizamos más en el ámbito de la educación popular, sin embargo pueden producirse muchos ámbitos de experiencias sociales, no solamente desde el trabajo social, sino que cualquier disciplina tiene una práctica, y esa práctica debería posibilitar producir conocimiento desde su práctica particular.

RP: ¿Podría afirmarse que la sistematización de experiencias, paulatinamente, fue surgiendo y se fue cimentando como una propuesta necesaria para entender y abarcar de una manera más adecuada las realidades complejas de América Latina? Es decir, como una contrapropuesta a otros modelos pedagógicos, si se 
pueden denominar así, que venían de otras partes del mundo, del llamado Norte Global, Europa, Estados Unidos, etc.

OJH: Hay una afirmación de que nuestra realidad tiene características propias y que, por tanto, de esas características debemos producir un conocimiento que sirva para mirar críticamente y para transformar nuestra propia realidad. Dentro de todo ese movimiento, que en realidad es el movimiento que marca el desarrollo de las ciencias sociales en América Latina en el período de 19601970, acontece el surgimiento de las ciencias sociales y la misma teoría de la dependencia, la mirada de la Sociología Crítica, empiezan a aparecer los elementos de la Teología de la Liberación, la Sociología y la Psicología de la Liberación. Son elementos que van marcando confluencias de pensamiento, donde un punto central es nuestra realidad, una realidad propia, particular y desde nuestra realidad debemos tener capacidad para producir conocimientos y producir teoría desde nuestra práctica. No significa negar aportes teóricos de otros contextos, pero una cosa es dialogar con esos aportes teóricos desde nuestra propia reflexión teórica, ya marcada por nuestro contexto, y otra cosa es copiar los modelos, los esquemas, las categorías, los conceptos creados en Europa, en Estados Unidos, que eran predominantes y que todavía siguen siendo en algunas disciplinas los modelos de referencia. Uno mira a veces tesis o trabajos donde prácticamente no existe ninguna referencia a una bibliografía latinoamericana y solo se utilizan autores "famosos" de otro contexto. Eso no está mal, no estoy negando eso, sino que no hay un vínculo de ese tipo de reflexión más universal con la capacidad de producción-conocimiento desde nuestra propia realidad.

RP: ¿Qué tanto ha influido la sistematización de experiencias como modelo de reflexión crítica surgido en América Latina, hacia esas otras latitudes como Europa o los Estados Unidos?

OJH: Hay un elemento a tomar en cuenta. Efectivamente la parte específica de sistematización desde experiencias de sistematización popular, ha cobrado cada vez más interés en los países del Norte Global, particularmente en dos sectores: uno, en la gente que hace trabajos de educación, trabajos de organización, trabajos de solidaridad, gente que está vinculada con América Latina y encuentra que ahí hay una fuente importante para construir un conocimiento, también ellos desde su propia realidad o desde ese vínculo que tienen organizaciones del Norte con los países del Sur Global. Otro, en la gente que está interesada más bien en cambiar los esquemas y los modelos de producción de conocimiento clásicos y que, por ejemplo, entran en la perspectiva de la investigación-acción o investigación-acción participativa. Este proceso en 
América Latina es claramente impulsado por Orlando Fals Borda, ${ }^{1}$ con la idea que él plantea en la década de 1970 sobre desarrollar un nuevo paradigma de construcción del conocimiento desde las Ciencias Sociales y que en ese proceso debe haber un diálogo de saberes, una construcción del conocimiento entre los actores sociales y las personas académicas y los científicos. En ese diálogo de saberes se va a producir un conocimiento que está vinculado con la acción transformadora. Ahí hay un hilo de vinculación con corrientes de la antropología, a nivel del Norte, que ya postulaban la idea que toda investigación debería estar vinculada con una acción. Lo que hace Fals Borda es añadirle una perspectiva de investigación-acción participativa; aunque ya había un movimiento casi mundial, en la década de 1980, de redes internacionales de investigación-acción o de investigación-acción participativa, con distintos nombres y distintas modalidades. Es gente que busca otras epistemologías, una manera de producir conocimiento y de producir categorías propias, desde las propias realidades, y encuentran en la perspectiva de investigación-acción o investigación-acción participativa o investigaciónparticipativa, que serían los tres tipos de modalidades confluyentes, una perspectiva que le da sentido social y político a la investigación científica, y que no le quita el valor científico, sino al contrario, se lo refuerza. Porque, precisamente, el conocimiento científico aparece como un conocimiento vinculado a realidades cambiantes, y no simplemente a la repetición de esquemas o de categorías que ya están preestablecidos, sino que hay que irlos recreando a partir de las distintas realidades.

En los últimos años pensadores como Boaventura de Sousa Santos, ${ }^{2}$ que también entra con el tema de la ecología de saberes, empiezan a hablar de que efectivamente inciden en las corrientes epistemológicas de las Ciencias Sociales dos elementos. Hay una limitación que tenemos con las categorías actuales, con las categorías que usábamos para entender los fenómenos actuales, por ende, hay que recrear las categorías. Por otro lado, lo que él llama el epistemicidio, es decir, hay unas propuestas epistemológicas que niegan otros saberes; los saberes cosmológicos, los saberes ancestrales de los pueblos originarios, muchas veces han sido totalmente depreciados. Se trata, por ende, de la idea de generar un diálogo de saberes y una ecología de saberes donde el conocimiento científico y el conocimiento popular, estén siempre en interacción. Esto más que pensarlo como una técnica, hay que vislumbrarlo como una propuesta filosófica.

\footnotetext{
1 Pensador y sociólogo colombiano, cuyos postulados indagaron en el compromiso político de la sociología en beneficio de los sectores populares desde los postulados de la "investigaciónacción-participativa".

2 Académico portugués, experto en el campo de las ciencias sociales, con especialidad en las áreas transdisciplinarias del derecho, la sociología, la economía y la epistemología. Sus obras han influido en el pensamiento social crítico contemporáneo, rompiendo con las teorías posmodernas y profundizando en la crítica radical de los enfoques hegemónicos con el rescate de conceptos tales como la emancipación y la interculturalidad.
} 
Es decir, el conocimiento y la relación entre los saberes, tiene que ver con las realidades, y ahí volvemos otra vez a Paulo Freire. ${ }^{3}$ Freire decía que tendríamos que conocer primero el mundo y luego lo nombramos. Entonces, toda la propuesta de alfabetización o de educación liberadora de Paulo Freire parte del hecho de que vivimos en un mundo, conocemos ese mundo, tenemos una comprensión porque estamos situados históricamente, pero necesitamos generar la capacidad de nombrarlo y, por lo tanto, el esfuerzo de reflexión teórica supone partir de esa realidad. Entonces, hay una confluencia de todos estos movimientos epistemológicos: lo que viene desde el trabajo social con la producción de un conocimiento sistemático de la práctica, lo que viene de la educación popular como producción de conocimiento a partir de la realidad para transformarla, lo que viene de la investigación-acción o investigación participativa como nuevo paradigma de las Ciencias Sociales. Procesos del teatro del oprimido, por ejemplo, de Augusto Boal, ${ }^{4}$ también en la década de 1960, tienen mucha fuerza. Boal dice que el teatro tiene que ser un reflejo de las contradicciones de la realidad. Entonces, más que ser una obra de un autor o una autora que simplemente lo piensa y lo coloca en esa escena, lo que hay que hacer es escenificar y dramatizar las situaciones de opresión en las que vivimos en la vida cotidiana. En el campo de la comunicación, por ejemplo, Mario Kaplún, ${ }^{5}$ empieza a cuestionar el modelo unidireccional de comunicación tradicional en el cual hay un emisor y unos receptores que reciben eso que se emite. Kaplún sostiene que las personas, cualquier persona, tiene que considerarse no solamente receptora de lo que recibe de los medios de comunicación, sino que debe considerarse también emisora, y por lo tanto, la voz de la gente tiene que ser incorporada en los medios de comunicación.

Tomando en cuenta esto, otro ejemplo que procede de estos procesos de crítica epistemológica propiamente latinoamericanos es la Teología de la Liberación. La Teología de la Liberación parte de que, por ejemplo, en lugar de que tomemos la Biblia, que fue escrita hace unos cuatro mil años, y la repitamos y tratemos de acomodar esas palabras a la realidad actual, más bien lo que hay que hacer es relacionar una lectura de la realidad que dialogue con el conocimiento, digamos en este caso, con las tradiciones bíblicas. En esa Teología de la Liberación, una de las propuestas es trabajar con comunidades, trabajar a partir del ver, juzgar y actuar, un método muy utilizado en

\footnotetext{
${ }^{3}$ Pedagogo brasileño con grandes aportes al pensamiento y práctica de la Educación Popular. Se le atribuye ser el promotor de la escuela de la Pedagogía Crítica y fuerte opositor de la educación bancaria, un concepto creado por él para criticar a la educación tradicional.

${ }^{4}$ Fue un dramaturgo, escritor y director de teatro brasileño, conocido por el desarrollo del Teatro del Oprimido. El cual es un método y formulación teórica de un teatro pedagógico que hace posible la transformación social.

${ }^{5}$ Fue un comunicador, radialista y escritor argentino, conocido por promover el concepto de la comunicación transformadora en oposición a la comunicación bancaria (educación bancaria de Freire).
}

Revista Perspectivas: Estudios Sociales y Educación Cívica - № 18 ISSN-L: 2215-4728 • Enero - Junio, 2019 • pp. 1-18 http://dx.doi.org/10.15359/rp.18.3 
comunidades cristianas en ese período cuando se crean las comunidades de base. Por lo tanto, hay que ver la realidad que estamos viviendo, analizarla, juzgarla, y luego actuar. De nuevo, el vínculo práctica-teoría y teoría-práctica, produce conocimientos desde la experiencia vivida. Sea desde una perspectiva religiosa, sea desde una perspectiva científica, sea desde una perspectiva pedagógica, se producen confluencias, y en esas confluencias la sistematización de experiencias desde la perspectiva de la educación popular, se ubica, se inserta con una propuesta engarzada con todo este movimiento.

RP: ¿Cuánto aporta la sistematización de experiencias en los procesos de investigación?

OJH: Bueno, yo pienso que sobre todo hay un aporte cualitativo porque la sistematización de experiencias son procesos de producción de conocimiento que los realizamos las propias personas que desarrollamos la experiencia. Hay formas de investigación donde no necesariamente las personas que vivimos las problemáticas producimos el conocimiento. Digamos que hay formas de investigación documental, hay formas de investigación que utilizan la observación participativa, que utilizan los focus group, etc., pero hay una persona que produce el conocimiento y la interpretación, a partir de esas informaciones. En la sistematización de experiencias no, la sistematización de experiencias es siempre participativa; lo que aporta principalmente es que nos damos cuenta que somos sujetos productores de conocimiento, y que además colectivamente podemos producir un conocimiento. Entonces, hay como un doble movimiento, es una producción colectiva de conocimiento que tiene como resultado la producción de un conocimiento colectivo -que es siempre provisional en el sentido que refleja una reflexión a partir de una realidad-, pero como esa realidad es cambiante y ese conocimiento debe servir para una mirada crítica, transformadora de la realidad, a la vez plantea un nuevo desafío. Lo decimos a veces en los talleres de sistematización de esta manera: vamos a hacer un proceso de sistematización en estos tres o cuatro meses, pero cuando terminemos, ya no vamos a ser las mismas personas, ya nosotras y nosotros vamos haber logrado mirar de repente cosas que antes no mirábamos, vamos a estar desafiados por lo que hemos descubierto, lo que existe en nuestras prácticas, vamos a tener otros elementos, conceptos, categorías creadas por el proceso colectivo y, por lo tanto, ya nuestra experiencia, que era nuestro punto de partida, ya quedó enriquecida por el propio proceso de sistematización.

Por lo tanto, tendríamos que continuar construyendo nuevos conocimientos a partir de la nueva experiencia que hemos vivido. Y ahí tal vez resaltaría un punto en relación con esto del cuánto aporta, que un proceso de sistematización puede que aporte más que el propio producto, porque a veces la gente dice, "queremos hacer una sistematización porque queremos un libro con 
tal cosa". Bueno perfecto, el libro es un producto, pero el proceso de lo que vamos construyendo el conocimiento, va a ser cualitativamente tal vez más importante por lo que implicó de ejercicio, de reflexión crítica, que al final, a veces en el libro, en la publicación de la producción que hagamos al final, no va a caber toda la riqueza de lo que significó que un grupo durante tres o cuatro meses hiciera un proceso de reflexión crítica.

RP: Entonces, ¿la sistematización de experiencias supera su propio fin?

OJH: Yo creo que sí, principalmente ese es uno de los valores principales, porque es un proceso colectivo, es un proceso crítico, nos volvemos, por lo tanto, más cuestionadores, más críticos, con más elementos, y claro que hay productos que deben surgir. A veces se piensa solo en el libro o el folleto, pero puede producirse una obra de teatro, puede producirse un audiovisual, un video, se pueden narrar los testimonios y producir un programa de radio. Es decir, los procesos de sistematización, al recopilar y reflexionar sobre diversos saberes, experiencias, anécdotas, los procesa, nos da un universo de posibilidades de materiales para producir muchas cosas. Sería muy estrecho si solo pensamos en la producción de un texto escrito, que sí, tal vez hay que hacerlo y la gran mayoría de las sistematizaciones con las que nosotros trabajamos produce también un texto escrito, que son informes de esa sistematización. Pero lo que digo es que no se agota en el informe, sino que hay muchísimos productos que podemos rescatar.

RP: Pensamos que uno de los aportes de la sistematización de experiencias se asemeja mucho a lo que, por ejemplo, Ezequiel Ander-Egg6 llama de "animación sociocultural" y el papel que podría asumir el animador sociocultural, en este caso, en contextos en los que está trabajando con una comunidad en específico, para intentar abordar determinado problema social, pero que ese problema social, que está afrontando una comunidad, sea abordado mediante el diseño de un proyecto o diagnósticos participativos. Es decir, que tomen en cuenta la experiencia vivida de las personas, como un conocimiento tan viable y tan importante como el propio conocimiento científico, en una relación dialéctica.

OJH: Efectivamente, sí hay muchos puntos de vinculación de la animación sociocultural. La visión de Ezequiel Ander-Egg vincula procesos de trabajo comunitario, procesos participativos, en función de proyectos específicos. Precisamente, por eso utiliza el término de la animación, el papel del educador o la educadora, como animador o animadora de un proceso colectivo. Desde esta óptica es básicamente lo mismo que ocurre con el proceso de educación popular:

6 Filósofo, sociólogo y ensayista argentino, destacado por sus obras en el Trabajo Social con insumos teóricos y prácticos a este campo.

Revista Perspectivas: Estudios Sociales y Educación Cívica - № 18 ISSN-L: 2215-4728 • Enero - Junio, 2019 • pp. 1-18 http://dx.doi.org/10.15359/rp.18.3 
el educador o la educadora no son personas que transmiten su saber, su conocimiento al resto de la gente que no lo saben, sino que va dinamizando un proceso de construcción colectiva de conocimiento. Yo diría que hay una coincidencia en cuanto al proceso metodológico participativo y colectivo, hay una coincidencia también en la medida que estamos partiendo de situaciones concretas de proyectos específicos en los cuales estamos involucrados, y que a veces tenemos nombres distintos para cosas que son muy similares, pero que son reflejo de distintas corrientes de pensamiento o de distintas tradiciones o trayectorias que se han ido formando.

Pero el concepto de animación sociocultural es un concepto creado, me parece en Europa, y que es muy extendido ahí en países como España que tiene muchos programas de animación sociocultural. Incluso, tengo entendido que hay hasta carreras en las cuales la gente estudia y se forma como animador sociocultural. Hay muchas confluencias de ese tipo de aproximación que creo que vienen de distintas maneras de nombrar o entender cosas que al final van siendo bastante confluyentes. Ahora bien, yo por eso no me preocupo mucho del uso de algunos términos, porque por ejemplo, hay gente que dice: esto que ustedes llaman sistematización, nosotros lo llamamos evaluación interpretativa. Perfecto, no tengo ningún problema, si lo que estamos haciendo es eso mismo, no hay problema. En cambio, lo importante es estar conscientes de lo siguiente: ¿qué estamos haciendo?, ¿cómo lo estamos haciendo?, ¿para qué lo estamos haciendo? Entonces, sistematización de experiencias primero es un proceso, es un enfoque, y es una propuesta metodológica que parte participativamente de los propios sujetos que viven la propia experiencia, son los principales sujetos protagonistas de la sistematización. Segundo, la sistematización no es para reconstruir solo lo que se ha vivido, sino para iluminar lo que queremos hacer. Por lo tanto, a veces se dice que es para apropiarnos del pasado, pero es principalmente para apropiarnos del futuro, o sea, para poder tener pistas hacia adelante. El tercer elemento propio de una sistematización de experiencias como nosotras y nosotros lo entendemos, es que siempre se hace una reconstrucción histórica de los procesos vividos, porque es una interpretación que supone reconstruir históricamente lo que se ha hecho, una toma de distancia que nos permita mirar críticamente aquello en lo que hemos participado. La sistematización de experiencias le da una importancia muy grande al cómo hemos vivido las experiencias y, por lo tanto, toda nuestra subjetividad está involucrada. Existen unas corrientes más positivistas que dicen: "si incorporamos lo subjetivo entonces estamos deformando el conocimiento". Pero lo subjetivo siempre está presente, aunque usted diga que no quiere tenerlo, porque está tomando una opción y porque está diciendo, "hay que hacerlo de esta manera". Por lo tanto, hay una intencionalidad en la acción. Además, se plantean aspectos fundamentales de una experiencia, que responde a la pregunta, ¿cómo lo vivimos?: si lo hicimos porque teníamos desesperación, o esperanza, o rabia, o frustración... 
RP: O por obligación.

OJH: O por obligación o lo que sea. Cuál fue nuestra actitud, nuestra emocionalidad en los procesos puede ser de repente el factor principal que explique si algo funciona o no funciona. Nuestras acciones están siempre marcadas, primero, de toda nuestra historia y todo el conjunto de contexto que hace que hagamos eso. Que lo hagamos de determinada manera, que lo hagamos con una persona, que lo hagamos con determinada sensibilidad e intencionalidad y emocionalidad. Por lo tanto, recoger la riqueza que está presente en lo que hacemos es mucho más complejo que si simplemente se hace un listado de actividades o hacer una suma de resultados medibles -que sí hay que hacerlos-. El hecho de que existan indicadores o datos medibles es parte de un proceso de sistematización pero no se reduce a eso. Hay una valoración de la dimensión educativa, porque al final la dimensión subjetiva es un factor fundamental de la transformación.

$\mathrm{Si}$ queremos hacer un proceso de cambio, cualquier proceso de transformación va a significar una disposición subjetiva transformadora, que en realidad es parte de cualquier proceso de producción de conocimiento. $\mathrm{O}$ sea, nunca conocemos de forma pasiva, no existe la transferencia unilateral de conocimiento, el profesor que viene y tira su "rollo" a los alumnos que escuchan, eso no es producción de conocimiento, en la medida en que lo que el profesor o la profesora la coloquen como información. En la medida en que esa información se relacione con otras informaciones y con los conocimientos que la gente tiene y que, por lo tanto, los correlacione con sus respectivas experiencias personales o colectivas, en esa medida yo produzco activamente mi proceso de conocimiento. Paulo Freire criticaba mucho la educación bancaria, en el sentido de que es como un banco donde tú depositas conocimiento, y el conocimiento se va acumulando, entonces hoy día digo $\mathrm{A}$, y mañana digo $\mathrm{B}$ y $\mathrm{C}$, etc... Pero esto no es cierto: el conocimiento siempre es un proceso activo de construcción.

Por ende, ¿cuál es la tarea del docente o de la docente en un proceso educativo de educación popular o de una sistematización de experiencias? Ayudar a generar ese proceso activo, a hacerlo consciente, hacerlo crítico y por lo tanto, en ese movimiento la persona que lo impulsa, el educador o la educadora también está aprendiendo. O sea, no vamos nunca solamente a ser educadores, vamos a ser siempre sujetos del aprendizaje que queremos generar. Por ejemplo, yo trabajo mucho en talleres de sistematización que en realidad son talleres donde no soy yo el que sistematiza, sino que estoy apoyando o asesorando metodológicamente a que la gente sistematice su experiencia. Al hacerlo, yo estoy enriqueciéndome de cómo están mirando su experiencia, de cómo la analizan, de qué resultados van obteniendo, de los debates que uno genere y, por lo tanto, después de cada sesión, uno obtiene una riqueza enorme de todo lo que ha salido del proceso. Es mucho más que 
enseñanza, supera la rutina, el trabajo burocrático de quien viene y repite su clase que todos los años la ha repetido igual, sino que cada clase o cada sesión es una aventura creadora, es una oportunidad de construir algo nuevo y es un desafío. Nosotros mismos vamos dinamizando y encontrando temas, preocupaciones, dilemas, cuestionamientos que de repente no teníamos antes de esa sesión; ahí está la riqueza de los procesos de sistematización de experiencias y dentro de esta perspectiva de educación popular.

RP: Es muy interactivo. Podríamos decir que la SE es válida para procesos de investigación, ya sea de investigación-acción, investigación-acción participativa o que contemplen alguna perspectiva aplicada a la sistematización de experiencias.

OJH: Puede perfectamente vincularse, no la contradice. Por ejemplo, vamos a trabajar en una comunidad, en un barrio popular y ahí vamos a trabajar con grupos de jóvenes, hombres y mujeres, y un tema que les interesa a ellos es poder rescatar cómo se constituyeron como un grupo cultural que trabaja teatro, danza y música, y quieren hacer un proceso de sistematización de su experiencia. Entonces, el punto de arranque de la sistematización de experiencias es esa experiencia personal y grupal de cómo ese grupo se fue creando, sus motivaciones, qué fue lo que los llevó a hacer esto, etc. Pero imaginémonos que ese proceso con ese grupo se inserta en un proyecto más amplio que quiere hacer un diagnóstico de la problemática juvenil en esa zona y, por lo tanto, quiere hacer un proceso de investigación-acción, entonces esos mismos jóvenes pueden participar haciendo un proceso de definir objetivos, de definir categorías y de hacer un estudio de la problemática en que vive ese barrio, en relación con la política juvenil y pueden utilizar técnicas de todo tipo -entrevistas, encuestas, focus group- como procesos de investigación-acción, porque eso los va a nutrir como equipo. Supongamos además que esto se enmarca en un proyecto de una universidad que quiere hacer un estudio demográfico sobre la situación de los barrios de tal zona. Bueno, entonces ese trabajo más particular, de ese grupo, que a la vez los involucra a ellos y ellas en esa investigación-acción participativa, podría ser un elemento que se combina con un estudio que podría ser El Estado de la Nación.

Cada esfuerzo investigativo de producción de conocimiento va a alimentar a los demás, y por eso lo importante es tener la posibilidad de nutrirse mutuamente de esos esfuerzos. Yo creo que sistematización de experiencias aporta algo, pero no aporta todo, al igual que un proceso de investigación-acción aporta algo, pero no lo aporta todo, o un estudio de investigación documental o un estudio más tradicional, más global, aporta algo, pero no lo aporta todo. Pero debe siempre estar presente la pregunta, ¿quién se apropia de eso?, o sea, ¿quién logra hacer ese engarce?, porque muchas veces está el proceso de la apropiación de esos conocimientos por parte de una 
entidad externa, puede ser una universidad, un centro de investigación, puede ser una persona investigadora que luego publica su tesis sobre todo lo que hubo ahí y es una persona que se apropió del conocimiento, lo sacó y lo puso. Pero otra cosa es que todo esto sirviera para fortalecer al movimiento juvenil de barrios y que se inserte dentro de esta estrategia. Es ahí donde estamos pensando que estos procesos de educación popular vinculan procesos de investigación, procesos de sistematización de distinto tipo, pero en función de fortalecer la capacidad protagónica de los sujetos populares.

RP: Es decir, la sistematización de experiencias no debería entenderse como una mera metodología, o técnica, que por ejemplo un científico social decida aplicar en su tesis de investigación con determinada comunidad, porque la comunidad no sería ya un agente pasivo, un mero participante, sería protagonista del conocimiento, del proceso de construcción del conocimiento, para atender determinada realidad.

Usted mencionó la crítica que se le hace al positivismo porque no considera viables los conocimientos, las experiencias vividas de las personas en las comunidades. Pero también sobresale la crítica hacia el narrativismo. Los positivistas más bien critican a los que toman en cuenta esas experiencias y manifiestan que no les están aplicando la debida crítica, que todo lo que están diciendo las personas es cierto.

OJH: Creo que es un debate válido cuando nos preocupamos sobre la validez del conocimiento que producimos. El problema es que no existe una validez en sí en general, sino una validez que responde a las preguntas, ¿para qué?, ¿para quién?, ¿a favor de qué?, ¿a favor de quién?, y por lo tanto, ¿en contra de qué? y ¿en contra de quién? No existe la producción de un conocimiento neutro o aislado de las condiciones. Es decir, retomando el ejemplo, si un grupo juvenil hace un proceso de sistematización y se relaciona con un proyecto de investigación que está teniendo una universidad y a la vez con una política del Estado que tiene que ver con el grupo juvenil, eso ¿a quién le va a servir?, o sea, ¿va a servir para manipular más al grupo de jóvenes, para poderlo tener amarrado políticamente hacia los intereses de quien está impulsando eso o va a servir para que tenga un protagonismo mayor, mayor criticidad? Siempre el tema de qué conocer y cómo conocer, está relacionado con el para qué conocer, para quién conocer, a favor de quién o de qué, por lo tanto, en contra de qué o de quién conocer. En realidad, esta es una frase de Paulo Freire: no existe la neutralidad del proceso. La pregunta es válida, lo que creo es que hay que tener una pluralidad epistemológica. Por ejemplo, la narración personal tiene un valor, pero no tiene tampoco un valor absoluto, pero tiene un valor en cuanto para mí, que lo estoy narrando, es una verdad que yo estoy construyendo. Ahora, que no es una verdad absoluta y general, sino que es en lo que yo me estoy afirmando, y puede ser que yo esté narrando algo que yo viví, pero como 
lo estoy narrando ahora, lo estoy narrando en función de lo que me interesa ahora rescatar de lo que viví, esto es, lo que está marcado por mi interés hoy.

Eso ocurre mucho en el tema de la historia oral. Creo que ese tipo de conocimientos tiene el valor en la medida de que nos muestra qué es lo que la gente en este momento está valorando de lo que fue su experiencia pasada, y si esa narración es puramente memorística, o sea, no está basada en un registro documental, sino es que te cuenta la historia de vida; cuándo empezamos en este barrio, cuándo empezamos acá y un grupo de gente..., toda esa historia es una historia que estamos reconstruyendo ahora, pero que no necesariamente está narrando lo que ocurrió en ese momento, porque no estás teniendo una referencia documental que te muestre ese proceso, se está usando solo la memoria. ¿Cuál es el valor de eso? Para mí, el valor es que eso es para la gente, que en este momento le interesa lo que pasó, que puede que incluso sea distinto de lo que pasó; que de repente es lo que le hubiera gustado que pasara, tiene el valor que esa persona le está asignando a ese tipo de intervención y en un proceso de sistematización de experiencias, en realidad esas narraciones son más de tipo anecdotario, que ayudan a ilustrar esto que estoy presentando, pero para una sistematización, nosotros tenemos que ir hacia la documentación del proceso. Por eso necesitamos tener registro. Yo no animo a que hagamos sistematizaciones basadas en la memoria, porque nuestra memoria es traicionera, nuestra memoria es interesada. Nosotros tocamos una parte, no sé cuánto... 10\% de lo que hemos vivido porque es la cosa que nos interesó más, o lo que odiamos más, o lo que fuera.

RP: La memoria es selectiva.

OJH: Siempre es selectiva. Nos damos cuenta si incluso nosotros tratáramos de reconstruir lo que hicimos en esta semana, a partir de nuestra memoria, estoy seguro que se nos olvida el $80 \%$ de las cosas que hicimos, o más. Pero si nosotros hubiéramos llevado todo anotado cada minuto o cada 10 minutos de lo que estamos haciendo durante la semana, y después registramos y miramos la cronología de lo que hemos hecho, empezamos a darnos cuenta que nos levantamos, que tomamos ese día un café y otro día un gallopinto, otro día que no tomamos huevo porque el doctor dijo que teníamos colesterol y que luego salimos temprano porque había una reunión, y que esa reunión era con quién y qué aprendí en esa reunión. Si yo tuviera todo esto anotado, yo lo puedo reconstruir, eso es lo que queremos hacer en la sistematización. O sea, hacer registros y reconstruir el proceso basados en los registros, y al hacerlo nos vamos a dar cuenta, primero, que hay muchas más cosas de las que nos acordamos. Segundo nos vamos a dar cuenta que hay un flujo de vínculos entre los distintos acontecimientos y podemos empezar a hacer interrelaciones, pero en un proceso de una organización o de un grupo que esté haciendo una sistematización, si se logran agarrar las actas de las reuniones que se tuvieron,

Revista Perspectivas: Estudios Sociales y Educación Cívica - o 18

ISSN-L: 2215-4728 • Enero - Junio, 2019• pp. 1-18

http://dx.doi.org/10.15359/rp.18.3 
si se tiene un registro de las fotografías que se tomaron, si están grabadas las intervenciones que la gente dijo en tal momento, si tienen anotado el horario con que las realizaron, recoger eso nos va a dar una riqueza enorme.

Entonces, todos vamos a tener una especie de espejo, con una cierta distancia de lo que hemos vivido, y entonces podemos empezar a mirar críticamente. Un grupo social que quiere sistematizar su experiencia de los últimos seis meses, si no tiene registros, lo que va a llegar al final es a acordarse de las cosas más sobresalientes, pero difícilmente va a poder sistematizar los detalles y matices de lo que pasó en esos seis meses. Nosotros proponemos trabajar siempre sobre registros y complementariamente utilizar la narración oral de memoria, y ojalá hacerla colectiva. Continuando el ejemplo, si todas las personas que participaron en esa actividad -o varias- empiezan a hacer una narración de la historia oral, entonces hay una triangulación y una manera de ir construyendo, por lo menos, una historia colectiva con distintos protagonistas que la vivieron.

No niego el valor de la narración personal, ya expliqué un poco el porqué me parece importante trabajar narraciones colectivas, pero me parece que para una sistematización de experiencias la rigurosidad que implica supone trabajar sobre los registros, supone trabajar sobre la documentación, que tiene que ser hecha durante la experiencia, si no es muy difícil tener una mirada crítica, vamos a tener una mirada más complaciente o una mirada más justificadora de lo que hicimos, porque en el fondo estamos aquí y en el fondo recojo lo que me ha servido para estar aquí. Pero no voy a mirar críticamente todas las cosas que no hice o que pude haber hecho y no hice porque las fui dejando de lado.

RP: Pero la sistematización de experiencias no se queda entonces, como usted nos lo ha explicado con anterioridad, en el simple hecho del registro. Tiene una intencionalidad transformadora de esa realidad en la que está inmerso el proceso.

OJH: Es descubrir los aprendizajes a partir de la reconstrucción de lo que tenemos en los registros. A partir de los registros vamos a ir reconstruyendo las etapas, los momentos de la experiencia que queremos sistematizar. Hay una intencionalidad, ¿para qué quiero sistematizar?: "me interesa porque quiero hacer un nuevo plan de trabajo y entonces quiero sistematizar cómo trabajamos en este año en esta zona". El producto, el resultado de esa sistematización va a ser una propuesta de trabajo para el próximo año. Hay una intencionalidad y no es simplemente el registro ni es simplemente la narración.

RP: La sistematización de experiencias podría emplearse como una perspectiva para poder leer de una mejor manera la memoria colectiva. No solo desde el punto de vista de lo que se toma en cuenta en el proceso de construcción de esa experiencia, sino también de lo que no se tomó en cuenta, de lo que no se dijo de 
los silencios o los silenciamientos de las personas. El registro nos permite ver también eso. Lo que se ocultó, lo que se cayó, y los posibles porqué de eso, y si deberíamos prestar atención o no a ese silencio.

OJH: Exactamente. En los registros aparecen continuidades, pero aparecen también discontinuidades, aparecen rupturas. Es mirar la experiencia desde una lógica de proceso, y los procesos son muy variados y complejos. A veces no nos damos cuenta de cómo hemos ido cambiando a lo largo del proceso por distintas circunstancias: "que fue que se nos ocurrió otra cosa, que vino alguien y nos propuso tal cuestión, alguien propuso y nadie le hizo caso, entonces no se hizo, yo propuse tal cosa y dijeron, jah sí!...”. Ese tipo de reacciones o comportamientos nosotros normalmente no los vamos registrando porque nos parecen irrelevantes en primera instancia. Cuando sí los registramos vamos a darnos cuenta del trayecto de las decisiones que tomamos, ante los distintos momentos y escenarios. Los silencios, las ausencias, las rupturas, las discontinuidades, son elementos explicativos del proceso que una sistematización nos permite vislumbrar con criticidad. Ahí es donde miramos críticamente por qué dejamos de hacer esto: "mirá, aquí veníamos haciendo tal cuestión y se trabajó tal tema y el tema fue una continuidad... y luego el tercer evento ya no lo hicimos y en el cuarto pasamos a trabajar tal cosa y en el quinto vino fulano e hizo tal cuestión y se quedó perdido".

RP: O no vino nadie a la reunión.

OJH: ¡Claro! "Y nuestro plan era que vinieran 50 personas a tal cosa, y resulta que vinieron 10. Entonces, ¿por qué vinieron 10 ?... y a la siguiente sesión vinieron 20". Bueno, ahí hay algo que me tiene que explicar por qué a la primera sesión vinieron 10 y a la siguiente 20, o al revés, vinieron 30 a la primera sesión y a la segunda 5. Entonces, ¿qué pasó en esa primera sesión?, ¿qué explica ese cambio? Eso es lo fascinante, empezar a encontrar los hallazgos que la propia experiencia nos muestra, que es lo que en la metodología de la sistematización nosotros llamamos "dejar hablar" a la experiencia. Reconstruimos para que la experiencia empiece a hablarnos e interrogamos a la experiencia. Es como un diálogo crítico donde un colectivo se enfrenta a la práctica realizada y construye un aprendizaje en función de una propuesta futura. Es un ejercicio seperapasionante. No es tan simple, pero tampoco es algo reservado al superespecialista. Como propuesta metodológica hay que procurar la rigurosidad suficiente para el tipo de registros, cómo utilizarlos y para poder explicitar los aprendizajes.

RP: ¿Cuáles podrían ser las potencialidades o limitaciones de la sistematización de experiencias aplicada a la experiencia pedagógica de aula, en la escuela, en el colegio, en una universidad? 
OJH: Primero, sobre la potencialidad de las experiencias que yo conozco, hay algo muy interesante que es cuando un grupo de docentes, o sea, no a un nivel individual, sino un grupo de docentes de un mismo centro educativo o de una universidad, establece sesiones de reflexión crítica sobre la metodología que está trabajando. Puede haber un eje común, que tiene que ver con el vínculo de los temas que trabajamos con los conocimientos de los estudiantes o con la realidad que está pasando en este momento en el país. Empezar a ver cómo cada uno y cada una de los docentes hemos trabajado eso o cuáles han sido los aprendizajes en términos de producción de material didáctico nuevo.

Por ejemplo, hace unos años, en Desamparados se hizo un proceso de sistematización interesante a través de un festival de innovaciones. Cada una y cada uno de los docentes trajo un poco de elementos que había creado a partir de darse cuenta que los materiales que usaba en sus clases eran insuficientes para lo que querían hacer. Entonces hubo mucha gente que había creado innovaciones didácticas o incluso algunas innovaciones de tipo más pedagógico. Es muy interesante, porque ese intercambio desde la práctica de los docentes, permite una reflexión tanto para quien quiere mostrar lo que hizo -porque tengo que justificar por qué lo hice así-, qué es lo que yo he descubierto al usar esto, qué recomendaciones yo haría sobre mi experiencia, y si yo hago eso, estoy también cualificando mi propio saber de lo que quería producir. La persona o los otros docentes que están escuchando y viendo mi experiencia, me van a empezar a hacer preguntas que de repente yo no me he hecho y cuando cada uno de los demás exponga sobre su vida, yo me voy a hacer preguntas y voy a conocer. Puede llegar a ser un intercambio enormemente rico si, por ejemplo, pudiéramos tener sesiones de formación docente, por decirle cada mes o cada dos meses, donde a partir de un eje de interés común, desde la práctica de los y las docentes, se pudieran tener sesiones de reflexión crítica. Serían pequeños ejercicios de minisistematización, porque no estaríamos hablando necesariamente de largos procesos de sistematización, que también puede ser, pero digamos esa sería una de las potencialidades; el aprendizaje mutuo, de los distintos saberes, de las distintas inquietudes.

Con respecto a las limitaciones, todo va a depender del alcance que tengan las experiencias. A veces hay pretensiones de querer sacar una sistematización de experiencias muy particulares, como una conclusión de largo alcance, o sea, yo no puedo a partir de mi enseñanza de matemáticas, decir: "basado en mi práctica pedagógica, voy a hacer una propuesta de política de la enseñanza de la matemática en el país”. Sería como extrapolar excesivamente. Pero si hiciéramos un amplio proceso de sistematización de experiencias de enseñanza de las matemáticas en la educación secundaria de este país, y ese trabajo de sistematización encontrara limitaciones, las posibilidades, las dificultades, las innovaciones, y rescatáramos eso de manera estructurada, de repente sería muy importante para que el famoso problema de los exámenes de

Revista Perspectivas: Estudios Sociales y Educación Cívica - № 18 ISSN-L: 2215-4728 • Enero - Junio, $2019 \bullet$ pp. 1-18 http://dx.doi.org/10.15359/rp.18.3 
bachillerato, la resistencia de las matemáticas, etc., pudieran superarse y pudieran dar una política educativa, pero nutrida de experiencias educativas que lo hagan posible.

Diría que el riesgo o la limitación principal es querer extrapolarlo. La otra limitación sería que yo encuentre conclusiones, o que encontremos perspectivas interesantes que aplicar y que no las hagamos, o sea, que sigamos repitiendo lo mismo pese a que hemos descubierto que hay nuevas potencialidades, por temor, por inseguridad, por muchas razones. Prosiguiendo el ejemplo anterior, decía de las innovaciones didácticas y pedagógicas que hubo en Desamparados. Para alguna gente era la primera vez que las mencionaba, porque decían que si los supervisores o las supervisoras se enteraban, los iban a sancionar porque se estaban saliendo del programa. Un elemento que podría ser muy valorado que es la capacidad innovadora del docente o de la docente, termina siendo frenado por una política sancionatoria, que podría tener el Ministerio, en lugar de una política de fomento de las innovaciones. Es algo que se podría incentivar de manera crítica y estructurada.

RP: Eso ayudaría desde el Ministerio de Educación Pública a un proceso de sistematización de experiencias con el fin de generar insumos necesarios para reestructurar los programas de estudio.

OJH: Claro. Que es lo que muchas veces no ocurre. No hay un diálogo desde la base de los procesos, que alimente a quienes definen las políticas. Esto lo podemos hablar en políticas de salud, políticas agropecuarias, políticas de educación, de vivienda, de lo que sea. Existen núcleos técnicos que elaboran políticas que son las que se ejecutan, pero la gente que las ejecuta, que está en el terreno cotidiano, poniéndolas en práctica, se va a dar cuenta de qué funciona, de qué no funciona y que muchas veces se inventan cosas para que funcionen, se dejan de lado algunas, pero nadie les pregunta. Nadie de los que elaboran los materiales o diseñan las políticas, se retroalimenta de lo que está ocurriendo.

Generar procesos de sistematización a nivel docente para alimentar las políticas del Ministerio de Educación, generar procesos de sistematización de promotoras y promotores de salud de la gente que atiende en los Equipos Básicos de Atención Integral en Salud (EBAIS) para alimentar las propuestas del Ministerio de Salud o de la Caja Costarricense de Seguro Social. Pero si eso no está pensado de esa manera, ni se lleva a registros, ni las personas sienten que se valora lo que se está haciendo, ni hay retroalimentación y, por lo tanto, las políticas se repiten y van fallando en lo mismo porque nunca hubo la posibilidad de transformarlas. 
RP: Entonces, más que considerar eventuales limitaciones intrínsecas en la sistematización de experiencias, más bien deberíamos enfocarnos en las características o limitaciones que la sistematización de experiencias puede encontrar en determinados contextos institucionalizados. Como lo son, por ejemplo, las propias universidades, sean públicas o privadas. Las universidades tienen una propia política de extensión para poder trabajar con comunidades, pero eventualmente existe un límite institucional que los extensionistas no pueden traspasar. Imaginemos si esos extensionistas deciden aplicar o proponerle a una comunidad evacuar la problemática desde el punto de vista de la sistematización de experiencias, pero que la propia política universitaria o institucional, no le permita trascender o no le permita transformar porque resulta peligroso o contraproducente para determinado interés político o económico.

OJH: Eso ha cambiado bastante, porque por ejemplo, una de las áreas en la que nosotros como ALFORJA trabajamos más es precisamente en esa, en la asesoría de las universidades. Estamos trabajando con la Universidad Nacional (UNA), estamos trabajando con la Universidad de Costa Rica (UCR), específicamente con programas de extensión cada año. Por ejemplo, esto que contaba de estos talleres de sistematización y metodología que estamos haciendo con investigadores/investigadoras para docentes. Primero hemos trabajado con un programa interuniversitario del Consejo Nacional de Rectores (CONARE), después asesoramos tres procesos o cuatro procesos de formación a extensionistas de la UNA. Con la UCR, se ha coordinado con la Vicerrectoría de Acción Social un trabajo bastante permanente y con mucho interés por parte de quienes están en las vicerrectorías de acción social o de extensión de cada universidad. Esto ocurre también en otros países de América Latina. Precisamente en estos días vengo de estar haciendo un taller en Brasil, otro taller en Argentina, otro taller en Uruguay y ahora otro taller aquí en Costa Rica. Son talleres diferentes pero al mismo tiempo aplican y reflexionan sobre la metodología de la sistematización de experiencias en la extensión universitaria. Es uno de los campos que más se ha abierto a esta perspectiva y felizmente porque es un campo muy importante. Otro ejemplo, con la Fundación Omar Dengo habíamos trabajado para recuperar aprendizajes de informática educativa a cerca de 500 centros de educación primaria. Estas cosas que estamos mencionando son experiencias que ocurren pero que ojalá que ocurrieran más.

San José, Costa Rica

Septiembre, 2018 